\title{
Nerve and Behavioral Responses of Mice to Various Umami Substances
}

\author{
Masataka Narukawa, ${ }^{*}$ Kanako Morita, Masahide Uemura, Ryo Kitada, \\ Seong-Hee $\mathrm{OH}$, and Yukako HaYASHI ${ }^{\dagger}$ \\ Graduate School of Agriculture, Kyoto University, Gokasho, Uji, Kyoto 611-0011, Japan
}

Received May 23, 2011; Accepted July 26, 2011; Online Publication, November 7, 2011

[doi:10.1271/bbb.110401]

Food contains various taste substances. Among them, umami substances play an important role with regard to the perception of the taste of food, but, few studies have examined the taste characteristics of representative umami substances other than monosodium L-glutamate (MSG). By conducting mouse behavioral studies (the 48-h 2-bottle preference test and the conditioned taste aversion test) and assessing gustatory nerve responses, we investigated the taste characteristics of unique umami substances, including sodium succinate, L-theanine, betaine, and the enantiomer of MSG, D-MSG. Furthermore, we examined the synergy of umami with inosine $5^{\prime}$-monophoshate (IMP). In the case of the mice, sodium succinate had an umami taste and showed strong synergy with IMP. L-Theanine showed synergy with IMP but did not have an umami taste without IMP. In contrast, betaine did not have an umami taste or synergy with IMP. D-MSG might have weak synergy with IMP.

Key words: umami; synergy; gustatory nerve response; 48-h 2-bottle preference test; conditioned taste aversion test

Food intake is essential for biological activity in all organisms. The interest level of people for food is high because eating delicious foods is pleasurable. Food palatability is comprehensively determined on the basis of many factors, including taste, flavour, color, shape, texture, and temperature. ${ }^{1)}$ Among these, taste plays an important role in palatability.

There are various types of tastes. Complicated taste is formed by a combination of basic tastes. ${ }^{2)}$ Taste is classified into five basic categories: saltiness, sweetness, bitterness, sourness, and umami. Each taste quality plays a role in nutrition. Bitter often signifies the presence of poison in food, whereas sour indicates unripe or spoiled food. Animals instinctively avoid bitter and sour and instead prefer salty, umami, and sweet tastes, which signify the presence of minerals, energy, and proteins respectively.

Umami taste is stimulated by compounds containing amino acids, such as L-glutamate, and 5'-ribonucleotides, such as inosine $5^{\prime}$-monophosphate (IMP) and guanosine 5'-monophosphate (GMP). These compounds are presant as mono- or disodium salts in meats, vegetables, and dairy products. ${ }^{3,4)}$ When amino acids and $5^{\prime}$-ribonucleotides are both present in food, umami taste intensity is synergistically enhanced, and the umami taste threshold is dramatically lowered. ${ }^{5)}$ This characteristic taste-enhancing effect, umami synergy, is a hallmark of umami taste in the case of humans and some other mammals. ${ }^{6)}$ Thus umami has unique properties, but, most studies on umami taste involve representative umami substances such as monosodium L-glutamate (MSG), IMP, and GMP. Hence, we focused on four substances that have hardly been investigated: sodium succinate, L-theanine, betaine, and D-MSG.

Sodium succinate is found in shellfish such as clams. ${ }^{7)}$ L-Theanine ( $\gamma$-glutamylethylamide) is a glutamic acid analog found in tea. ${ }^{8)}$ Betaine (trimethylglycine) is found in many plants including beets, legumes, and fruits, and in animals such as lobsters, crabs, octopus, squid, and fish. ${ }^{9)}$ The free and bound forms of D-MSG, an enantiomer of MSG, are found not only in bacteria but also in plants, insects, and vertebrates. ${ }^{10,11)}$ In this study, we performed a behavioral assay and an electrophysiological experiment on mice in order to evaluate the taste characteristics of these four substances. In particular, we examined to determine whether they show synergy with IMP.

\section{Materials and Methods}

Reagents. MSG, sodium succinate, D-MSG, and betaine were purchased from Nacalai Tesque (Kyoto, Japan). IMP was from Sigma (St. Louis, MO). L-Theanine was from Taiyo chemical (Mie, Japan). All the other reagents were of analytical grade and were from standard suppliers.

Animals. The study population included female C57BL/6J mice (age, 7-20 weeks; Japan SLC, Hamamatsu, Japan). The animals were housed at 3-4 per cage at the Kyoto University Animal Care Facility. They were provided ad libitum access to standard mouse chow and tap water. Temperature and humidity were maintained at $21^{\circ} \mathrm{C}$ and 45-50\% with consistent 12-h light/dark cycles (lights switched on at 0700). All the experiments were performed following to protocols approved by the Kyoto University Animal Care Committee.

Forty-eight $h$ 2-bottle preference test. Twenty-four mice were assigned to 6 groups (four mice per cage). For $48 \mathrm{~h}$, they were presented with two bottles of $100-\mathrm{mL}$ capacity: one containing deionized water, and the other a tastant solution. After the $24 \mathrm{~h}$, the bottle positions were switched in order to control for positional effects. The ratio of tastant consumed to total liquid consumed was recorded for each tastant. For each tastant, the solutions were presented in an

\footnotetext{
$\dagger$ To whom correspondence should be addressed. Fax: +81-774-38-3749; E-mail: yhayashi@kais.kyoto-u.ac.jp

* Present address: German Institute of Human Nutrition Potsdam-Rehbruecke, Arthur-Scheunert-Allee 114-116, 14558 Nuthetal, Germany

Abbreviations: MSG, monosodium L-glutamate; IMP, inosine 5'-monophoshate; GMP, guanosine 5'-monophosphate; CTA, conditioned taste aversion; CS, conditioned stimulus; CT, chorda tympani; GL, glossopharyngeal
} 
A MSG

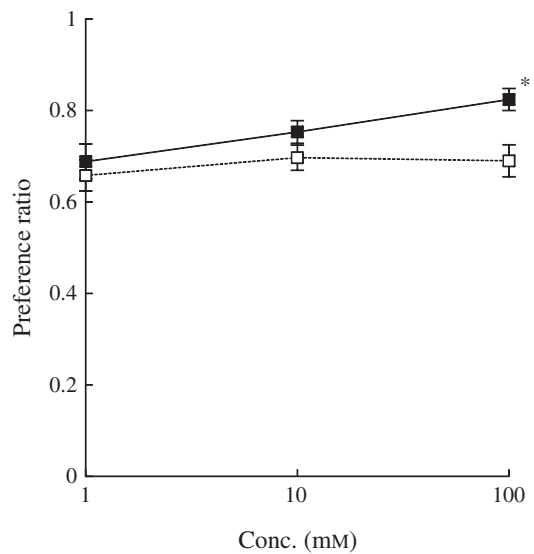

D D-MSG

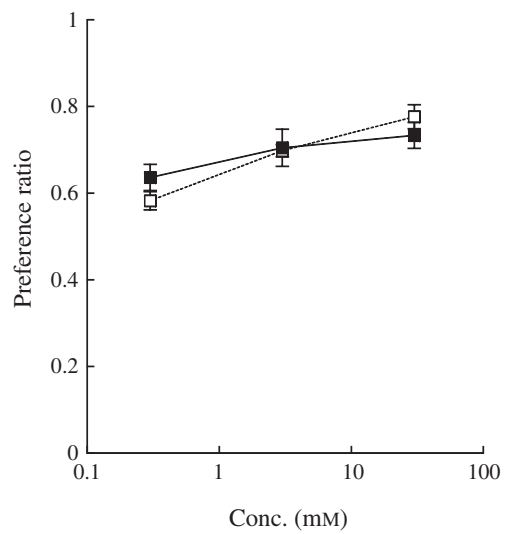

B Sodium succinate

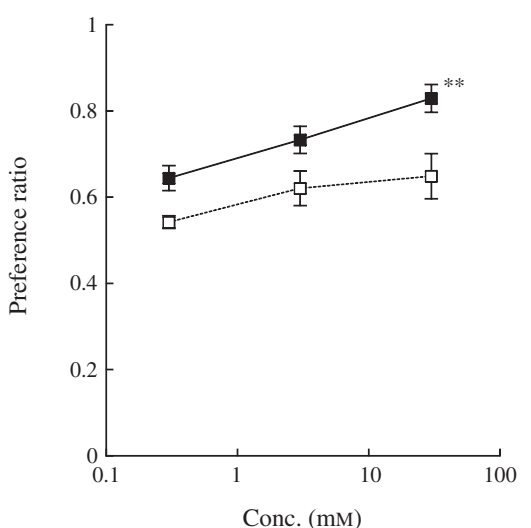

E Betaine

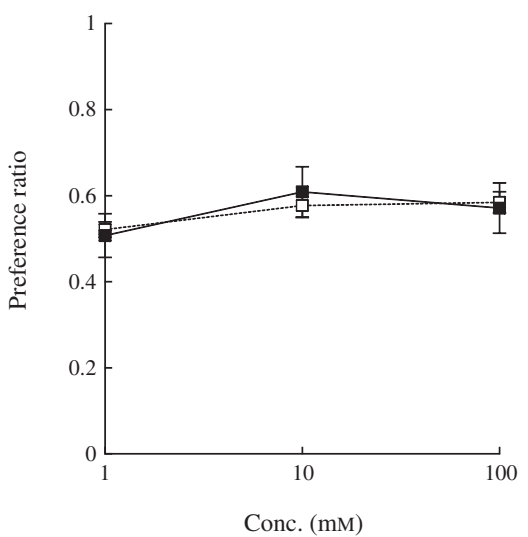

C L-Theanine

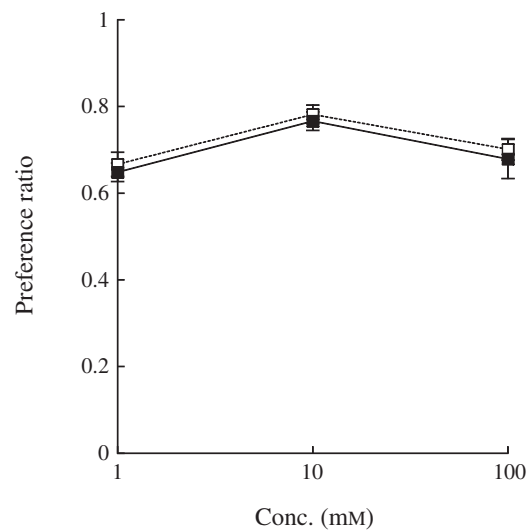

Fig. 1. Mean Preference Ratios for Umami Substances on the 48-h 2-Bottle Preference Tests.

A, MSG; B, sodium succinate; C, L-theanine; D, D-MSG; E, betaine. The white squares and broken lines indicate the tested substance used alone. The solid squares and unbroken lines indicate mixtures containing the substance tested and $0.5 \mathrm{mM}$ IMP. The preference ratio for $0.5 \mathrm{mM}$ IMP was $0.52 \pm 0.01$. Points represent the mean \pm SEM value. * and ${ }^{* *}$ indicate $p<0.05$ and $p<0.01$ respectively (2-way repeated ANOVA). For each group, $\mathrm{n}=6$.

ascending concentration series. Before the preference test, the mice were habituated by the presentation of two bottles that contained, only water for 1 week. When the different tastants were evaluated, new mouse groups were used. The tastants used for the 48-h 2-bottle preference test included 1,10 , and $100 \mathrm{mM}$ MSG; $0.3,3$, and $30 \mathrm{~mm}$ sodium succinate; 1,10 , and $100 \mathrm{~mm}$ L-theanine; $0.3,3$, and $30 \mathrm{~mm}$ D-MSG; 1, 10, and $100 \mathrm{~mm}$ betaine; and mixtures of these compounds with $0.5 \mathrm{mM}$ IMP.

Conditioned taste aversion (CTA) test. The mice were maintained on a $22 \mathrm{~h} / \mathrm{d}$ schedule of water deprivation. On the first training day, each animal was kept in a test box and given ad libitum access to distilled water through a single drinking tube for $1 \mathrm{~h}$. Supplemental water was available for $1 \mathrm{~h}$ in the home cage. From the second to the fifth day, the training time was reduced from $1 \mathrm{~h}$ to $10 \mathrm{~min}$. On the sixth day, each animal was given access to the mixture solution of $100 \mathrm{mM}$ MSG, $0.5 \mathrm{~mm}$ IMP, and $10 \mu \mathrm{M}$ amiloride as the conditioned stimulus (CS) for $10 \mathrm{~min}$, and then given an intraperitoneal injection of $0.15 \mathrm{M}$ $\mathrm{LiCl}$ ( $2 \%$ of body weight), which induces internal malaise with gastrointestinal distress, as the unconditioned stimulus. The control mice were injected with physiological saline instead of $\mathrm{LiCl}$ after ingestion of the CS. To reduce the taste response to $\mathrm{Na}^{+}$, the sodium channel inhibitor amiloride was added to the CS. ${ }^{12)}$ On the seventh day, a brief-access $(10 \mathrm{~min})$ 2-bottle preference test was performed. A total of 186 mice were used in the CTA test. The number of mice for a given treatment group was 6-12. A given mouse tried only one taste solution. The tastants used in the CTA test included $100 \mathrm{~mm}$ MSG, $100 \mathrm{~mm}$ sodium succinate, $300 \mathrm{~mm}$ L-theanine, $30 \mathrm{~mm}$ D-MSG, $300 \mathrm{~mm}$ betaine, and mixtures of these compounds with $0.5 \mathrm{mM}$ IMP
Gustatory nerve recordings. Whole-nerve responses to lingual application of the tastants were recorded from the chorda tympani (CT) and the glossopharyngeal (GL) nerves. Mice $(n=5-9)$ were anesthetized with an intraperitoneal injection of sodium pentobarbital $(50 \mathrm{mg} / \mathrm{kg}$ of body weight) and ethyl carbamate $(500 \mathrm{mg} / \mathrm{kg}$ of body weight). The anesthetic level was controlled by the additional injection of ethyl carbamate. A tracheal cannula was implanted, and the animal was secured using a head holder. The CT nerve was exposed at its exit from the lingual nerve by removal of the internal pterygoid muscle. The CT nerve was then dissected free of the surrounding tissues and cut at the point of entry to the bulla. The GL nerve was exposed by removing the digastricus muscle and the posterior horn of the hyoid bone. It was then dissected free from the underlying tissues and cut near the point of entry to the posterior lacerated foramen.

The exposed CT and GL nerves were placed on a platinum wire electrode. An indifferent electrode was positioned nearby in the wound. The whole-nerve activities were amplified, displayed on an oscilloscope, and monitored using an amplifier (DAM80, World Precision Instruments, Florida). The amplified signal was passed through an integrator with a time constant of $0.3 \mathrm{~s}$. The magnitude of the whole-nerve response was measured as the height of the integrated response from the baseline (before stimulation) approximately $5 \mathrm{~s}$ after the onset of stimulation to avoid the tactile effects of the stimuli. Tastants were applied for $30 \mathrm{~s}$, followed by a rinse with deionized water for $>30 \mathrm{~s}$. Each tastant concentration was presented at least 3 times, and the mean whole-nerve response was calculated. The relative response magnitudes, of tastants, were calculated taking the response magnitude for $100 \mathrm{mM} \mathrm{NH}_{4} \mathrm{Cl}$ as unity (1.0). The tastants used for the nerve recordings included 1,10 , and $100 \mathrm{~mm}$ MSG; 0.3, 3, and $30 \mathrm{~mm}$ 

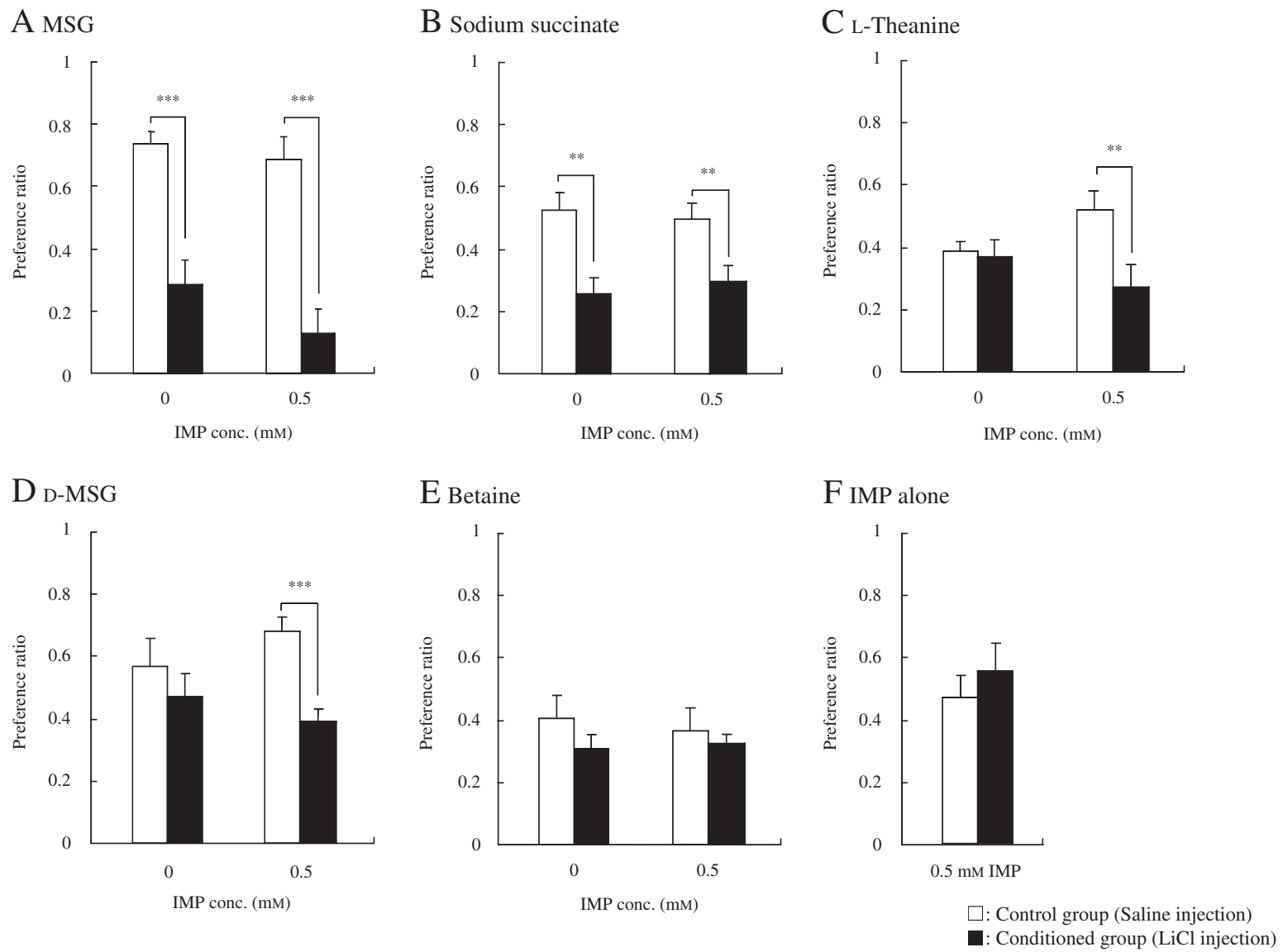

Fig. 2. Changes in Taste Quality Due to IMP Addition.

Mice that generalize umami (100 mM MSG + 0.5 mM IMP + $10 \mu \mathrm{M}$ amiloride) were used. A, MSG; B, sodium succinate; C, L-theanine; D, D-MSG; E, betaine; F, IMP alone. The white column and the black column indicate the control group mice, which were injected with saline, and the conditioned group mice, which were injected with $\mathrm{LiCl}$. The values for each column represent the mean $\pm \mathrm{SEM}$ values. ${ }^{*},{ }^{* *}$, and ${ }^{* * *}$ indicate $p<0.05, p<0.01$, and $p<0.001$ respectively (control group versus conditioned group, Student's $t$-test). For each group, $\mathrm{n}=6-12$.

sodium succinate; 1,10 , and $100 \mathrm{~mm}$ L-theanine; $0.3,3$, and $30 \mathrm{~mm}$ D-MSG; 1, 10, and $100 \mathrm{~mm}$ betaine; and mixtures of compounds of them with $0.5 \mathrm{~mm}$ IMP.

Statistical analysis. Data were expressed as mean \pm standard error of the mean (SEM). The statistical significance of differences was analyzed by Student's $t$-test and 2-way repeated measure analysis (ANOVA). Differences were considered significant at $p<0.05$. With regard to synergy, the response values for the mixtures of substances were compared with the response values with a mean IMP value added to the value for a given substance alone.

\section{Results}

Changes in the palatability of umami substances due to the addition of IMP

The preference ratios of the substances are shown in Fig. 1. The preference ratios of all the substances tested were over 0.5 at the concentrations used in the study. The mice preferred the test substances to water (Fig. 1A-D). The preference ratio for betaine was $0.5-$ 0.6 , lower than that for the other taste substances tested (Fig. 1E). The preference ratio for $0.5 \mathrm{~mm}$ IMP was $0.52 \pm 0.01$. The mice did not strongly prefer $0.5 \mathrm{~mm}$ IMP when used alone over water. When $0.5 \mathrm{mM}$ IMP was added to MSG and sodium succinate, the preference ratios increased synergistically (Fig. 1A and B). However, the preference ratios for L-theanine, D-MSG, and betaine did not increase even when IMP was added
(Fig. 1C-E). These results suggest that sodium succinate as well as MSG show synergy with IMP.

\section{Changes in taste quality due to IMP addition}

To determine whether these substances have an umami taste for mice, we performed a CTA test with substances with umami taste as CS. In the mice that generalized the umami stimulus (100 mM MSG +0.5 $\mathrm{mM}$ IMP $+10 \mu \mathrm{M}$ Amiloride), the preference for MSG and sodium succinate with and without addition of IMP significantly decreased as compared with the control mice (Fig. 2A and B). This suggests that $100 \mathrm{~mm}$ sodium succinate as well as $100 \mathrm{~mm}$ MSG had umami taste for the mice with and without IMP. With regard to $300 \mathrm{~mm}$ L-theanine and $30 \mathrm{~mm}$ D-MSG, no significant difference was observed between the conditioned and the control mice group with regard to preference for L-theanine alone and for D-MSG alone. However, the preference of the conditioned group for L-theanine and D-MSG significantly decreased when IMP was added (Fig. 2C and D). Thus, although these substances did not have obvious umami taste without IMP, they acquired umami taste due to IMP addition. In contrast, no significant difference was observed between these groups with respect to the $300 \mathrm{~mm}$ betaine solution with or without IMP addition (Fig. 2E). Hence, it is thought that $300 \mathrm{~mm}$ betaine did not have an obvious umami taste with or without IMP addition. No difference was observed 
A MSG

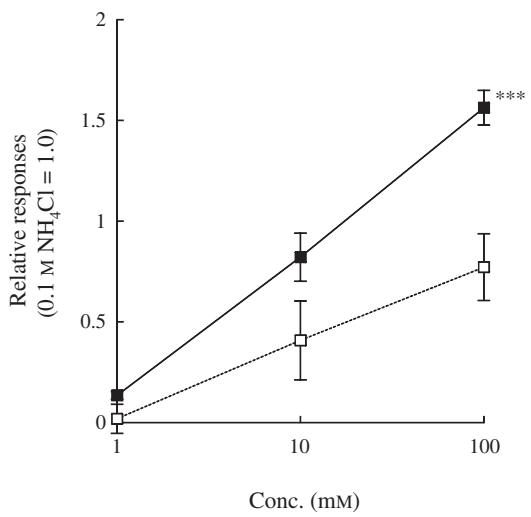

D D-MSG

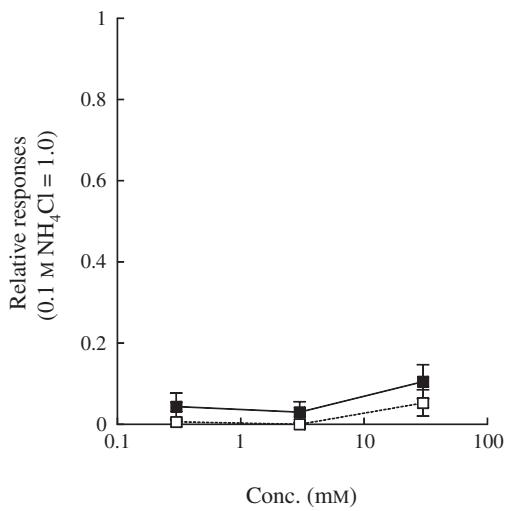

B Sodium succinate

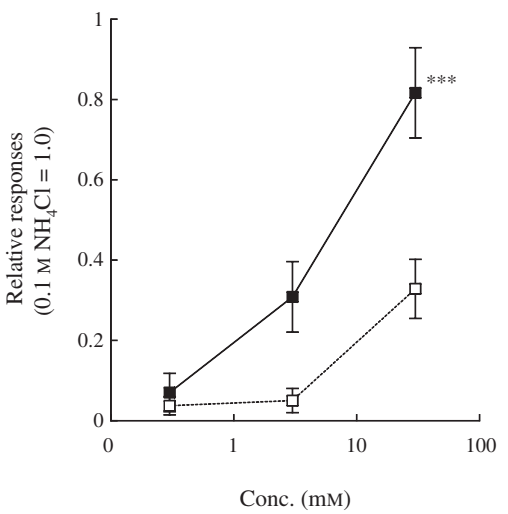

E Betaine

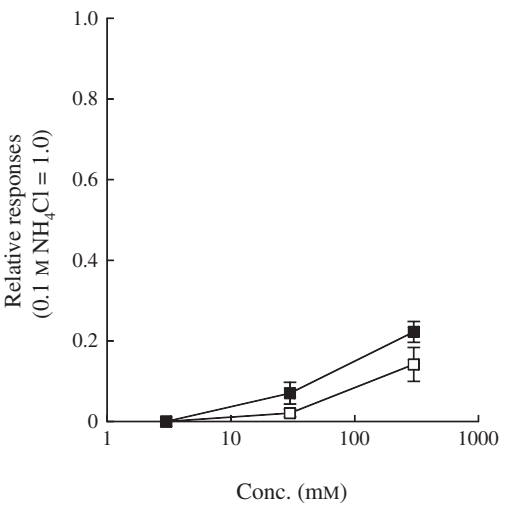

$\mathrm{C}_{\text {L-Theanine }}$

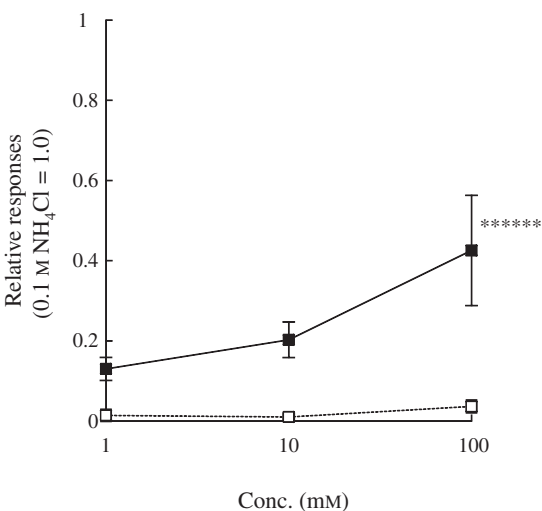

Fig. 3. Changes in CT Nerve Responses to Umami Substances Due to IMP Addition.

The integrated CT response for lingual application of tastants was normalized to the response for $100 \mathrm{mM} \mathrm{NH}_{4} \mathrm{Cl}\left(\mathrm{NH}_{4} \mathrm{Cl}\right.$ response $\left.=1.0\right)$. A, MSG; B, sodium succinate; C, L-theanine; D, D-MSG; and E, betaine. The hollow squares and broken lines indicate the tested substance used alone. The solid squares and unbroken lines indicate mixtures containing the tested substance and $0.5 \mathrm{mM}$ IMP. The CT response to $0.5 \mathrm{~mm}$ IMP alone was $0.01 \pm 0.01$. Points represent the mean \pm SEM value. ${ }^{* * *}$ and ${ }^{* * * * * *}$ indicate $p<0.001$ and $p<0.000001$ respectively $(2$-way repeated ANOVA). For each group, $n=5-6$.

between the groups with regard to preference for $0.5 \mathrm{mM}$ IMP (Fig. 2F). This suggests that L-theanine and D-MSG as well as MSG had synergy with IMP.

Assessment of synergy by using gustatory nerve recordings

In the CT nerve, the nerve responses to the substances tested increased in a concentration-dependent manner (Fig. 3). On the other hand, $0.5 \mathrm{~mm}$ IMP hardly induced CT nerve responses $(0.01 \pm 0.01)$. When $0.5 \mathrm{mM}$ IMP was added to MSG, sodium succinate, and L-theanine, nerve responses increased synergistically (Fig. 3A-C). When $0.5 \mathrm{~mm}$ IMP was added to D-MSG and betaine, nerve responses increased slightly, but, this increase was additive rather than synergistic (Fig. 3D and E). These results indicate that sodium succinate, L-theanine, and MSG have synergy with IMP.

In the GL nerve as well as in the CT nerve, responses to the substances tested increased in a concentrationdependent manner (Fig. 4). The GL nerve hardly responded to $0.5 \mathrm{~mm}$ IMP $(0.04 \pm 0.04)$. The GL nerve responses to MSG, sodium succinate, and L-theanine tended to be smaller than the $\mathrm{CT}$ nerve responses to these substances (Fig. 3A-C and Fig. 4A-C). In contrast, the GL nerve responses to D-MSG and betaine tended to be larger than the CT nerve responses for these substances (Fig. 3D and E and Fig. 4D and E). How- ever, no synergistic response was observed to the substances tested, even when 0.5 mM IMP was added.

Comparison of the brief-access 2-bottle preference test results for L-theanine and the L-theanine and IMP mixtures

Although L-theanine showed a synergistic response in the CT nerve recording (Fig. 3C), no difference was observed between the preference ratios for L-theanine alone and the mixtures containing L-theanine and IMP during the 48-h 2-bottle preference test (Fig. 1C). L-Theanine has various pharmacological effects. ${ }^{13)}$ Hence, to reduce post-digestion effects, we performed a brief-access 2-bottle preference test. Although there was no significant difference between $100 \mathrm{~mm}$ L-theanine and $100 \mathrm{~mm}$ L-theanine and the $0.5 \mathrm{~mm}$ IMP mixture, the mice strongly preferred the IMP mixture to L-theanine alone ( $p=0.09$, Fig. 5$)$. The preference ratio for $0.5 \mathrm{mM}$ IMP was $0.58 \pm 0.05$. This confirms the results for the CTA and CT nerve responses. We believe that the preference for L-theanine and/or its taste intensity increased with the addition of IMP.

\section{Discussion}

We investigated changes in palatability, taste quality, and taste intensity of umami substances using a 48-h 2-bottle preference test, a CTA test, and gustatory nerve 


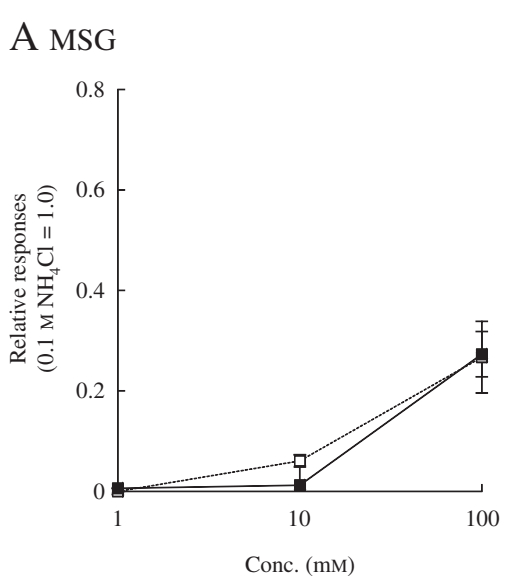

B Sodium succinate

C L-Theanine
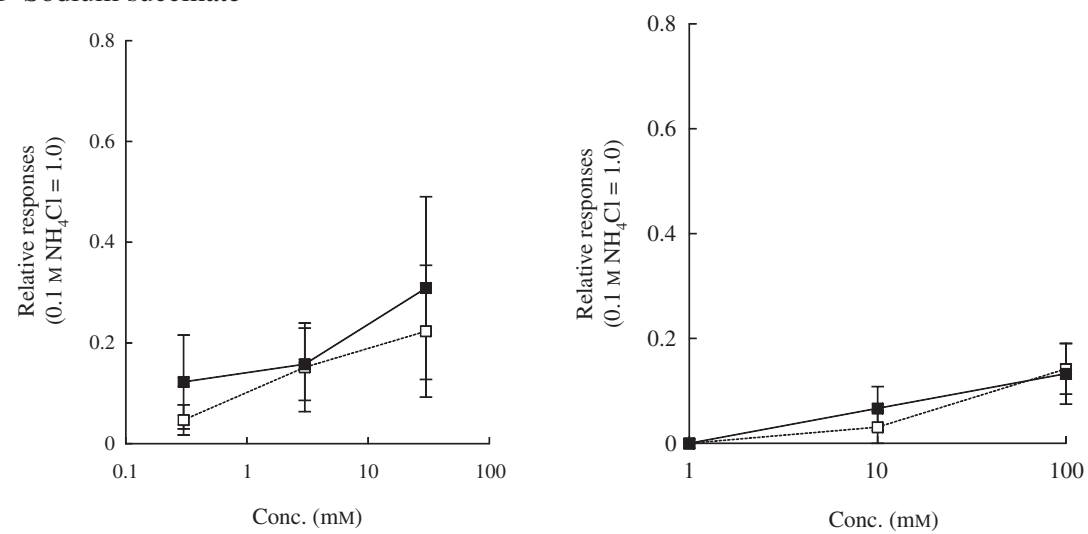

D D-MSG

E Betaine
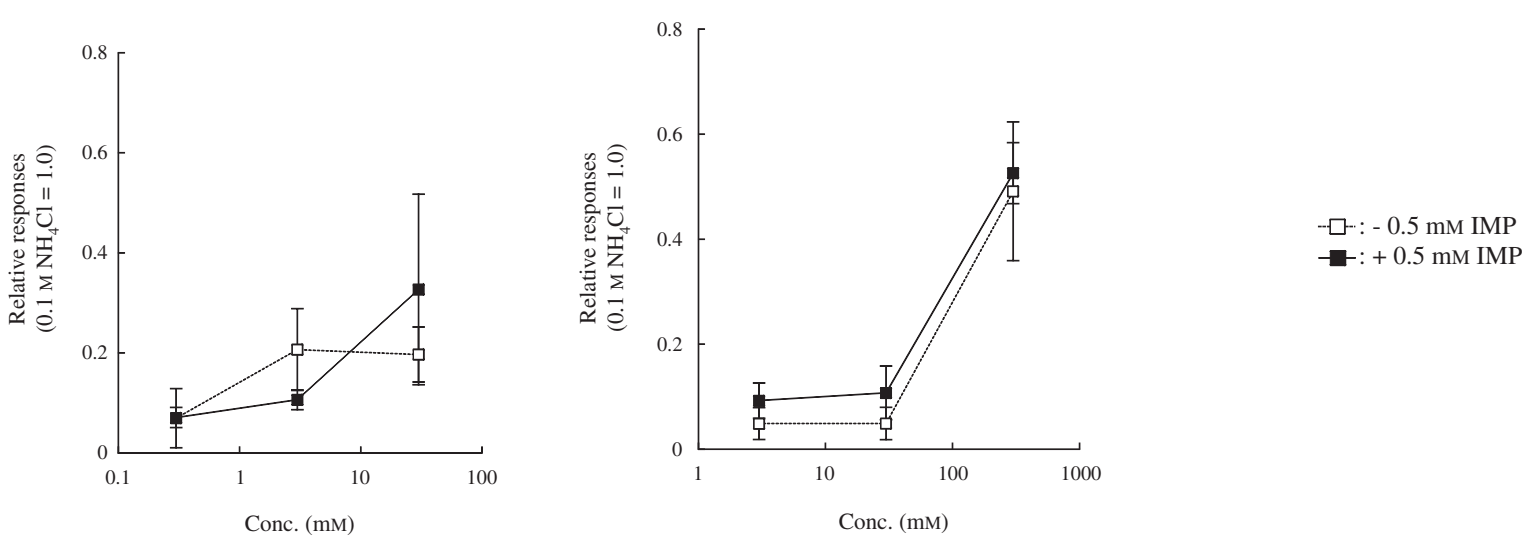

Fig. 4. Changes in the GL Nerve Responses to Umami Substances Due to IMP Addition.

The integrated GL responses for lingual application of tastants were normalized to the response for $100 \mathrm{mM} \mathrm{NH}_{4} \mathrm{Cl}\left(\mathrm{NH}_{4} \mathrm{Cl}\right.$ response $\left.=1.0\right)$. A, MSG; B, sodium succinate; C, L-theanine; D, D-MSG; E, betaine. The white squares and broken lines indicate the tested substance used alone. The solid squares and unbroken lines indicate mixtures containing the tested substance and $0.5 \mathrm{mM}$ IMP. The GL response to $0.5 \mathrm{mM}$ IMP alone is $0.04 \pm 0.04$. For each group, $n=5-8$. Points represent the mean \pm SEM value.

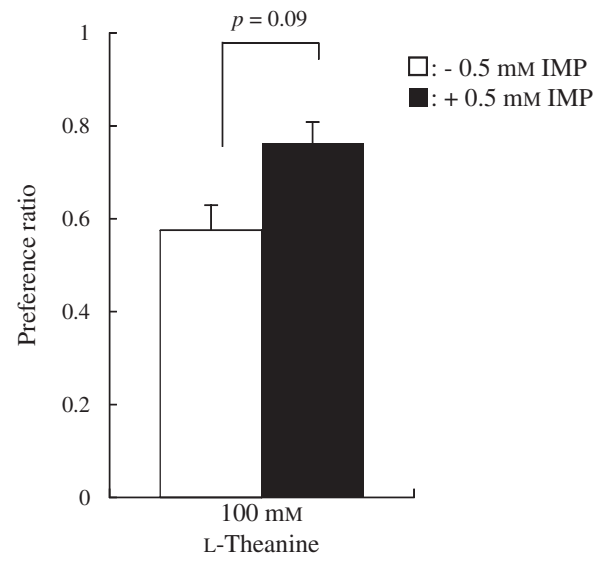

Fig. 5. Mean Preference Ratios for L-Theanine and the IMP Mixture of L-Theanine on the Brief-Access 2-Bottle Preference Tests.

The hollow column shows $100 \mathrm{~mm}$ L-theanine alone. The solid column shows $100 \mathrm{~mm}$ L-theanine $+0.5 \mathrm{~mm}$ IMP. Points represent the mean \pm SEM value. The preference ratio for $0.5 \mathrm{mM}$ IMP was $0.58 \pm 0.05$. For each group, $\mathrm{n}=8$.

recording respectively. We used MSG as a representative umami substance in addition to sodium succinate, L-theanine, betaine, and D-MSG. The results indicated that sodium succinate as well as MSG had umami taste for the mice and showed strong synergy with IMP.
L-Theanine showed synergy with IMP, but did not have umami taste without IMP. In contrast, betaine might not possess umami taste and synergy with IMP, and D-MSG might possess weak synergy with IMP.

Previous studies have identified three putative G protein-coupled receptors for umami substances: tastemGluR $1,{ }^{14)}$ taste-mGluR4, ${ }^{15,16)}$ and the amino-acid receptor T1R1 + T1R3 heterodimer. ${ }^{17,18)}$ Synergy, a hallmark of umami taste, was observed not only at the individual level but also at the cellular level. ${ }^{19,20)}$ Among these putative umami receptors, T1R1 + T1R3 is thought to be the main umami receptor, because it shows, a synergistic response between glutamate and 5 -ribonucleotides. Zhang et al. recently reported on the synergy mechanism for the T1R1 + T1R3 receptor. ${ }^{21)}$ They proposed a cooperative ligand-binding model involving the Venus flytrap domain of T1R1, where L-glutamate binds close to the hinge region and $5^{\prime}$ ribonucleotides bind to an adjacent site close to the opening of the flytrap to stabilize the closed conformation further.

Umami is a preferred taste, ${ }^{2)}$ and hence the mice preferred umami substances such as MSG. When umami substances like MSG have synergy with IMP, mice should prefer the IMP mixture over the umami substance alone on the 48-h 2-bottle preference test. All the tested substances were preferred by the mice. The 
preference ratio for sodium succinate clearly increased on the addition of IMP, but, the preference ratios for L-theanine, betaine, and D-MSG did not change on IMP addition. Because the preference ratio for MSG synergistically increased on the addition of IMP, it was possible that sodium succinate was responsible for the synergy.

Using the CTA test, we investigated the changes in taste quality caused by the addition of IMP. The umami taste intensity of umami substances like MSG becomes strong on the addition of IMP. Therefore, if synergy occurs, mice that generalize umami tastes avoid substances that have umami taste. Because the conditioned mice avoided MSG and sodium succinate in the presence and the absence of IMP, these substances were considered to have umami taste for the mice, regardless of IMP. In contrast, although the conditioned mice did not avoid L-theanine or D-MSG when used alone, they avoided the L-theanine and IMP mixture as well as the D-MSG and IMP mixture. Hence, we concluded that although L-theanine and D-MSG alone did not possess umami taste, they acquired umami taste on the addition of IMP. In addition, because there was no significant difference in the preference for betaine with and without the addition of IMP as between the conditioned and the control mice, we concluded that betaine does not have umami taste for mice, even when IMP is added. Although MSG and sodium succinate, expected to show a synergistic response with IMP in the control mice on the CTA test, the preference ratio for MSG and sodium succinate alone and, for the IMP mixture of them, was almost same. As one possible reason for this, because the preference ratio for the substances came to an upper limit, the difference between the substance alone and the mixture might not have been observable.

We measured gustatory nerve responses, which became strong when there was synergy. In the CT nerve recordings, the responses to sodium succinate and L-theanine increased drastically on the addition of IMP. In contrast, the responses to betaine and D-MSG did not change significantly when IMP was added. This suggests that L-theanine and sodium succinate also have synergy with IMP. However, on the 48-h 2-bottle preference test, synergy was observed in the case of sodium succinate but not L-theanine. To explain this discrepancy, we performed the brief-access 2-bottle preference test, and found that the mice strongly preferred the L-theanine and IMP mixture over Ltheanine alone. L-Theanine is known to have various pharmacological effects. ${ }^{13)}$ Due to post digestion effects such as absorption and metabolism, the difference might be not observable on the 48-h 2-bottle preference test.

Because the four substances that we used activated both the CT and the GL nerve, they could be detected in the entire tongue, but no increased response due to IMP addition was observed for the GL nerve, because almost all the T1R1 + T1R3 receptors, the receptors involved in synergy, were expressed in the anterior portion of the tongue, which is innervated by the CT nerve, ${ }^{22)}$ and electrophysiological analysis has shown that umami synergy occurs more prominently in the CT nerve than in the GL nerve. ${ }^{23)}$ Although further studies involving the heterologous cell expression system are required, these compounds might be detected by the T1R1+ T1R3 receptor.
With regard to D-MSG, although no synergistic increase in umami taste was observed on the 48-h 2-bottle preference test or the nerve recording assay, umami taste increased on the addition of IMP on the CTA test. Hence, we think that D-MSG may possess weak synergy with IMP. With regard to betaine, the response was greater in the GL nerve than in the CT nerve for $300 \mathrm{~mm}$ betaine. It is known that nerve responses to bitter substances are stronger in the GL nerve than in the CT nerve. ${ }^{24)}$ The taste of betaine at high concentrations might be perceived as bitter rather than umami. This hypothesis was confirmed by the results of the CTA test, which indicated that betaine did not have umami taste for the mice. Because the taste properties of betaine are unlike those of the other substances tested, it is possible that the receptive mechanism for betaine differs from that of the other substances tested.

We found that sodium succinate had synergy with IMP in the case of mice. This is interesting because succinate is an organic acid. Umami synergy is generally thought to occur between amino acids and $5^{\prime}$-ribonucleotides. ${ }^{5)}$ In addition, it is known that succinic acid does not have synergy with IMP in humans. ${ }^{25)}$ Thus, succinate can be used as a tool to investigate the differences in receptive mechanisms as between humans and mice.

\section{Acknowledgments}

This work was supported in part by a Grant-in-Aid for Scientific Research (C) (20580128) and by a Grant-inAid for Young Scientists (B) (21780131) in the Priority Area Food Science from the Japan Society for the Promotion of Science.

\section{References}

1) Sorensen LB, Moller P, Flint A, Martens M, and Raben A, Int. J. Obes. Relat. Metab. Disord., 27, 1152-1166 (2003).

2) Chandrashekar J, Hoon MA, Ryba NJ, and Zuker CS, Nature, 444, 288-294 (2006).

3) Ikeda K, J. Tokyo Chem. Soc., 30, 820-836 (1909).

4) Kodama S, J. Tokyo Chem. Soc., 34, 751-757 (1913).

5) Yamaguchi $\mathrm{S}$ and Kimizuka A, "Glutamic Acid: Advances in Biochemistry and Physiology,” eds. Filer LJ, Garattini S, Kare MR, Reynolds WA, and Wurtman RJ, Raven Press, New York, pp. 35-54 (1979).

6) Ninomiya $\mathrm{Y}$, Kurenuma S, Nomura $\mathrm{T}$, Uebayashi $\mathrm{H}$, and Kawamura H, Comp. Biochem. Physiol. A Comp. Physiol., 101, 97-102 (1992).

7) Konosu S, J. Food Sci. Technol., 20, $432-439$ (1973).

8) Sakato Y, Nippon Nôgeikagaku Kaishi, 23, 262-267 (1949).

9) Sakamoto A, Nishimura Y, Ono H, and Sakura N, Pediatr. Int., 44, 409-413 (2002).

10) Erbe $\mathrm{T}$ and Bruckner $\mathrm{H}$, J. Chromatogr. A, 881, 81-91 (2000).

11) Bruckner $\mathrm{H}$ and Westhauser $\mathrm{T}$, Amino Acids, 24, 43-55 (2003).

12) Kurihara K and Kashiwayanagi M, J. Nutr., 130, 931S-934S (2000).

13) Lu K, Gray MA, Oliver C, Liley DT, Harrison BJ, Bartholomeusz CF, Phan KL, and Nathan PJ, Hum. Psychopharmacol., 19, 457-465 (2004).

14) Toyono $T$, Seta $Y$, Kataoka S, Kawano S, Shigemoto R, and Toyoshima K, Cell Tissue Res., 313, 29-35 (2003).

15) Chaudhari N, Yang H, Lamp C, Delay E, Cartford C, Than T, and Roper S, J. Neurosci., 16, 3817-3826 (1996). 
16) Chaudhari N, Landin AM, and Roper SD, Nat. Neurosci., 3, 113-119 (2000).

17) Li X, Staszewski L, Xu H, Durick K, Zoller M, and Adler E, Proc. Natl. Acad. Sci. USA, 99, 4692-4696 (2002).

18) Nelson G, Chandrashekar J, Hoon MA, Feng L, Zhao G, Ryba NJ, and Zuker CS, Nature, 416, 199-202 (2002).

19) Narukawa M, Mori T, and Hayashi Y, Biosci. Biotechnol. Biochem., 70, 2613-2619 (2006).

20) Narukawa M, Kitagawa-Iseki K, Oike H, Abe K, Mori T, and Hayashi Y, Neuroreport, 19, 1169-1173 (2008).
21) Zhang F, Klebansky B, Fine RM, Xu H, Pronin A, Liu H, Tachdjian C, and Li X, Proc. Natl. Acad. Sci. USA, 105, 2093020934 (2008).

22) Kusakabe Y, Kim MR, Miura H, Shindo Y, Ninomiya Y, and Hino A, Chem. Senses, Suppl 1, i23-i24 (2005).

23) Sako N, Harada S, and Yamamoto T, Physiol. Behav., 71, 193198 (2000).

24) Danilova V and Hellekant G, BMC Neurosci., 4, 5 (2003).

25) Fuke S and Shimizu T, Trends Food Sci. Technol., 4, 246-251 (1993). 